\title{
QUASAR ABSORPTION LINES WITH A NONZERO COSMOLOGICAL CONSTANT
}

\author{
EDWIN L. TURNER \\ Princeton University Observatory, Peyton Hall, Princeton, NJ 08544 \\ AND \\ SATORU IKEUCHI \\ National Astronomical Observatory, Mitaka, Tokyo 181, Japan \\ Received 1991 July 8; accepted 1991 October 28
}

\begin{abstract}
Flat but nonzero cosmological constant model universes contain substantially larger comoving volumes and path lengths within a given redshift than more conventional zero $\Lambda$ ones. They also change the nature of their expansion at a characteristic critical redshift $z_{c}=\left(\lambda_{0} / \Omega_{0}\right)^{1 / 3}$, whereas zero $\Lambda$ flat models show a smooth power-law expansion at all epochs. Both of these physical differences can affect intergalactic absorption and scattering processes. The former simply implies more absorption for a given fixed population of absorbers or, conversely, requires fewer absorbers to explain a given observed amount of absorption. The latter manifests itself as a break in the number of absorbers per unit redshift at about $z_{c}$ and gives rise to a quite different redshift dependence of the number of absorbers at $z<z_{c}$. Thus, there is an interesting interaction between $\Lambda$ effects and cosmic absorption phenomena which are explored in this paper. For example, the absolute frequency of metal and high column density $\mathrm{H}_{\mathrm{I}}$ absorption at high redshift is significantly closer to that expected from the properties of low-redshift galaxies in $\Lambda$-dominated cosmologies than in conventional ones.

The equations describing IGM absorption statistics are developed for nonzero $\Lambda$ cosmologies, both for unevolving absorber populations and including the effects of the expected absorber evolution in some of the more popular physical models for the IGM and intergalactic clouds. These results are compared to observations for the cases of Lyman- $\alpha$ forest lines, damped Lyman- $\alpha$ lines, Lyman limit systems, C IV $1549 \AA$ lines, Mg II $2800 \AA$ lines, diffuse $\mathrm{H}$ I absorption (Gunn-Peterson effect), and the Compton $y$-parameter. Cosmological models with $\left(\Omega_{0}, \lambda_{0}\right)$ of $(1,0)$ and $(0.1,0.9)$ are contrasted. In general, the available data are consistent with both models, but the $(0.1,0.9)$ model extrapolates to much less absorption at low redshift and requires less evolution with redshift than the $(1,0)$ model. Hubble Space Telescope and ASTRO space UV spectroscopy of low-redshift absorption lines should allow a fairly direct test of $\Lambda$. The $\mathrm{Mg}$ II absorption line data currently extend to fairly low redshifts and allow the best A discrimination available with present data; the $(0.1,0.9)$ model gives a beter fit to the $\mathrm{Mg}$ II observations, but the size of the statistical errors is such that the $(1,0)$ model is not excluded with great significance. It is also found that the available Gunn-Peterson effect and Compton $y$-parameter limits are somewhat more difficult to satisfy in the $(0.1,0.9)$ model than in the $(1,0)$ one.
\end{abstract}

Subject headings: cosmology: theory — dust, extinction — intergalactic medium — quasars: absorption lines

\section{INTRODUCTION}

In recent years it has become clear that cosmological models in which the universe is currently dominated or strongly influenced by a positive cosmological constant $\Lambda$ merit further examination. The advantages of such models include easing the apparent conflict between globular cluster ages (Sandage \& Cacciari 1990) and the best recent determinations of the cosmic expansion time (Fukugita \& Hogan 1990; Tonry 1991; Jacoby, Ciardullo \& Ford 1990), helping explain the surprisingly large numbers of faint galaxies (Fukugita et al. 1990), more readily producing high-amplitude large-scale structure within the constraints imposed by the extreme isotropy of the cosmic microwave background (Kofman \& Starobinsky 1985; Bond 1990; Sugiyama, Gouda, \& Sasaki 1990), and allowing the possibility of reconciling the theoretical preference for a spatially flat universe with the typically small cosmic mean densities indicated by most observational measures (Trimble 1987). Some (Turner 1991) have gone so far as to declare $\Lambda$-dominated models a "best-fit" case. While these models are generally considered theoretically unappealing in various ways (Kolb \& Turner 1989), their only current empirical difficulty appears to be a tendency to predict too much gravitational lensing (Gott, Park, \& Lee 1989; Fukugita, Futamase, \& Kasai 1990a; Turner 1990), but when considered in detail (Fukugita \& Turner 1991), the lensing test does not yet appear to entirely exclude many interesting high- $\Lambda$ models.

With this motivation, we then examine in this paper a variety of intergalactic absorption phenomena in nonzero cosmological constant cosmologies. One of the important physical differences between zero and significantly nonzero $\Lambda$ models is that the latter contain far larger comoving volumes (or, equivalently, far longer comoving path lengths) within a given redshift. Indeed, this is a large part of the reason that they predict such vigorous gravitational lensing; there is simply a greater chance of having a lensing object near the line of sight along the increased path length. For the same reason, we expect that absorption processes will be strongly influenced by $\Lambda$ and therefore that observations of IGM absorption might well contain useful information or constraints on $\Lambda$-dominated cosmologies. There is another important physical effect which might also leave its signature in the observations; at a redshift of about $\left(\lambda_{0} / \Omega_{0}\right)^{1 / 3}$ where $\lambda_{0}=\Lambda c^{2} / 3 H_{0}^{2}$ and $\Omega_{0}$ is the usual cosmic mean density parameter, a $\Lambda$-dominated universe 
changes the basic character of its dynamical evolution. This may result in a change in the statistical properties of absorption systems above and below this characteristic redshift.

Many absorption lines are detected in quasars. They are valuable tools for probing intervening galaxies and the intergalactic medium (IGM). By using the observed quantities such as the equivalent width, Doppler parameter, observed number per unit redshift and so on, we can estimate the temperature, density, and size of absorbers as well as their space density. A most interesting point of absorption systems is that the observed redshift range covers $z \sim 0$ to $z \sim 4$ although the observed ions are different in various redshift (wavelength) regions. At present, we have absorption data on $\mathrm{Mg}$ II at $z=0.3-1.5$, on C IV at $z=1.0-3.0$, on Lyman limit systems (LLS) at $z=0.6-3.5$, on narrow sharp Lyman- $\alpha$ lines (Lyman- $\alpha$ forest) at $z=1.7-4.0$, and on damped Lyman- $\alpha$ lines at $z=1.7-3.5$. For these absorption systems, two important statistical results have been reported: one is the evolution of number density per unit redshift, and the other is the $\mathrm{H}$ I column density distribution. These data constrain the origins and the cosmological evolution of absorbers. In principle, we could extract information on the geometry of the universe from the observed number density of each type of absorption system, if we could distinguish the effects of evolution of each absorber from those of the cosmological model. Finally, the Gunn-Peterson test (absence of absorption) is also a potentially valuable probe of the curvature of the universe in addition to the $\mathrm{H}$ I density at $z=1.7-4.7$. Very recently, UV spectroscopy from spacecraft has provided Lyman- $\alpha$ forest data and a Gunn-Peterson limit at low redshift (Bahcall et al. 1991; Morris et al. 1991).

All the work on the absorption systems until now has been limited to cosmological models with zero cosmological constant, and the number density evolution is assumed to come from the evolution of size (or gas density), temperature, ionization state, and/or abundance in absorbers.

In the present paper, we examine quasar absorption lines in universes with a nonzero cosmological constant. As is well known, there are many uncertainties in the observational data concerning the number density per unit redshift and the $\mathrm{H}$ I column density distribution, which we will use in the present analysis. Also, we must presently take simple physical models for absorbers and crudely approximate evolution laws as powers of $1+z$. However, adequate or inadequate the models and simple characterizations of the data are, it at least allows a direct comparison of zero and nonzero cosmological constant cosmologies by following a similar approach in both cases.

In $\S 2$, we describe the basic physical effects and principles for the universe with a nonzero cosmological constant. In $\S 3$, we present a general discussion of various evolution effects for the absorbers and the observed number density evolution of each absorption system to discriminate between the evolution effects and cosmological model. In $\S 4$, a general discussion and several predictions and tests for a nonzero cosmological constant are presented, especially regarding space UV observations.

\section{BASIC PHYSICAL EFFECTS AND PRINCIPLES}

\subsection{Universe with a Nonzero Cosmological Constant}

The Einstein equations for the universe with a nonzero cosmological constant are written as

$$
\left(\frac{\dot{a}}{a}\right)^{2}=\frac{8 \pi G}{3 c^{2}} \rho-\frac{K c^{2}}{a^{2}}+\frac{\Lambda c^{2}}{3}
$$

and

$$
\frac{\ddot{a}}{a}=-\frac{4 \pi G}{3 c^{2}}(\rho+3 P)+\frac{1}{3} \Lambda c^{2},
$$

where $a(t)$ is the cosmic scale factor and other symbols have their usual meanings. The cosmological model is uniquely determined by two constants $K$ and $\Lambda$ and three time-dependent variables $a(t), \rho(t)$, and $P(t)$. These cosmological parameters are usually represented by their present values,

$$
H_{0} \equiv(\dot{a} / a)_{0}, \quad q_{0} \equiv\left(\ddot{a} a / \dot{a}^{2}\right)_{0}, \quad k_{0} \equiv K c^{2} / H_{0}^{2}, \quad \lambda_{0} \equiv \Lambda c^{2} / 3 H_{0}^{2}, \quad \Omega_{0} \equiv\left(\rho / \rho_{c}\right)_{0}=\left(8 \pi G \rho / 3 H_{0}^{2}\right),
$$

where the subscript 0 denotes the present value.

General expressions for the Hubble constant and deceleration parameter are

$$
\begin{gathered}
H(z)=H_{0}\left[(1+z)^{3} \Omega_{0}-(1+z)^{2} k_{0}+\lambda_{0}\right]^{1 / 2},\left(a_{0}=1\right), \\
q_{0}=\frac{\Omega_{0}}{2}-\lambda_{0},
\end{gathered}
$$

where we assume $\rho_{0} \gg P_{0}$. From equation (2.4), we can get the relation between the redshift and time

$$
t=-\frac{1}{H_{0}} \int_{z}^{\infty} \frac{1}{1+z} \frac{d z}{\left[(1+z)^{3} \Omega_{0}-(1+z)^{2} k_{0}+\lambda_{0}\right]^{1 / 2}} .
$$

In the following, we set $k_{0}=0$ (flat universe) as predicted by the inflation model and $\lambda_{0}>0$. Then we have

$$
\begin{aligned}
\left(H / H_{0}\right) & \sim \Omega_{0}^{1 / 2}(1+z)^{3 / 2} & & \text { at } z \gg z_{c}, \\
& \sim \lambda_{0}^{1 / 2} & & \text { at } z \ll z_{c},
\end{aligned}
$$


where the critical redshift $z_{c}$ is defined as

$$
1+z_{c} \equiv\left(\lambda_{0} / \Omega_{0}\right)^{1 / 3} \text {. }
$$

Therefore, at a large redshift $\left(z \gg z_{c}\right)$ the expansion law of the universe is the same as for the flat universe with zero cosmological constant but with a smaller effective Hubble constant, $H_{0, \text { eff }}=H_{0} \Omega_{0}^{1 / 2}$. As shown later, this affects the absorber density normalization at $z=0$. For a small redshift $\left(z \ll z_{c}\right)$, the universe is similar to a de Sitter universe, which is distinctly different from the dynamical evolution law of a zero-A universe.

\subsection{Expected Number per Unit Redshift}

The expected number of absorbers along a line of sight at redshift $(z, z+d z)$ is written as

$$
d N=\sigma_{a}(z) n_{a}(z)\left(\frac{c d t}{d z}\right) d z,
$$

where $\sigma_{a}$ and $n_{a}$ are the cross section and space density of absorbers. From equation (2.6) with $k_{0}=0$, we have

$$
\frac{d N}{d z}=\frac{c}{H_{0}} \frac{n_{a}(z) \sigma_{a}(z)}{(1+z)\left[(1+z)^{3} \Omega_{0}+\lambda_{0}\right]^{1 / 2}}
$$

At $z=0$, it becomes

$$
\left(\frac{d N}{d z}\right)_{0}=\frac{c n_{a}(0) \sigma_{a}(0)}{H_{0}} .
$$

Taking the mean values for nearby galaxies as $n_{a}(0)=10^{-2} n_{-2} \mathrm{Mpc}^{-3}, \sigma_{a}(0)=\pi R^{2}=100 \pi R_{10}^{2}(\mathrm{kpc})^{2}$, and $H_{0}=100 h \mathrm{~km} \mathrm{~s}$ $\mathrm{Mpc}^{-1}$, the expected number at $z=0$ is

$$
\left(\frac{d N}{d z}\right)_{0}=9.4 \times 10^{-3} h^{-1} n_{-2} R_{10}^{2} .
$$

This is the expected number of galaxy intersections per unit redshift at small redshift.

If the comoving space density of absorbers is conserved, $n_{a}(z)=n_{a}(0)(1+z)^{3}$, and the size is unchanged the expected number per unit redshift at $z \gg z_{c}$ becomes

$$
\left(\frac{d N}{d z}\right) \simeq \frac{c n_{a}(0)}{H_{0} \Omega_{0}^{1 / 2}} \sigma_{a}(0)(1+z)^{1 / 2} .
$$

This indicates that the redshift dependence $\left[\propto(1+z)^{1 / 2}\right]$ is the same as for the zero- $\Lambda$ flat universe but the volume is larger. In other words, it is equivalent to a universe with an apparently small Hubble constant, $H_{0 \text {, eff }}=H_{0} \Omega_{0}^{1 / 2}$, or an apparently larger space density $n_{a \text {, eff }}(0)=n_{a}(0) / \Omega_{0}^{1 / 2}$. The latter case will be discussed in relation to the Lyman limit systems and damped Lyman- $\alpha$ lines.

At $z \ll z_{c}$, the expected number per unit redshift becomes

$$
\left(\frac{d N}{d z}\right) \simeq\left(\frac{d N}{d z}\right)_{0} \frac{(1+z)^{2}}{\lambda_{0}^{1 / 2}}
$$

This shows a much steeper redshift dependence than zero- $\Lambda$ models.

In Figure 1, we illustrate the expected numbers per unit redshift for several cases of $\left(\Omega_{0}, \lambda_{0}\right)$ with $\Omega_{0}+\lambda_{0}=1$ and the case of $k_{0}=-1$ with $\Omega_{0}=\lambda_{0}=0$, for comparison. All are normalized at $z=0$ as in equation (2.12). Observations of damped Lyman- $\alpha$ systems LLS, Mg II, and C IV systems are also shown.

If $\Omega_{0}=1$ and $\lambda_{0}=0$, the observed numbers of LLS, Mg II, and C IV systems are about 100 times greater than that expected from galaxies of $h^{-1}=n_{-2}=R_{10}=1$, i.e., $h^{-1} n_{-2} R_{10}^{2} \sim 100$ for these absorption systems. On the other hand, the observed number of damped Lyman- $\alpha$ systems is consistent with $h^{-1} n_{-2} R_{10}^{2} \sim 10$. However, for an $\Omega_{0}=0.1, \lambda_{0}=0.9$ model the LLS, Mg II, and C IV absorber excess is a factor of 10, while damped Lyman- $\alpha$ systems are only a factor of 2 . Thus, in terms of a simple gross comparison of the observed frequencies of quasar absorption lines with that expected from the properties of present-day galaxies, $\Lambda$-dominated cosmologies considerably ease but do not entirely eliminate the long-standing discrepancy (Bahcall \& Spitzer 1969).

\subsection{Total Number of Absorbers}

Integrating equation (2.10) from $z$ to 0 for nonevolving absorbers, we have

$$
N(z)=\frac{2}{3 \Omega_{0}}\left(\frac{d N}{d z}\right)_{0}\left\{\left[\Omega_{0}(1+z)^{3}+\lambda_{0}\right]^{1 / 2}-1\right\} \sim 6.3 \times 10^{-3} h^{-1} n_{-2} R_{10}^{2} \Omega_{0}^{-1}\left\{\left[\Omega_{0}(1+z)^{3}+\lambda_{0}\right]^{1 / 2}-1\right\} .
$$

In this expression, the normalization factor depends upon $\Omega_{0}$, and we may renormalize it as $H_{0, \text { eff }}=H_{0} \Omega_{0}$ or $n_{a \text {, eff }}(0)=n_{a}(0) / \Omega_{0}$.

As two extreme cases, we present expressions for the $\Omega_{0}=1$ case and the $\lambda_{0}=1$ case as

$$
\begin{array}{rlrl}
\Omega_{0} & =1, & & N(z)=6.3 \times 10^{-3} h^{-1} n_{-2} R_{10}^{2}\left[(1+z)^{3 / 2}-1\right], \\
\lambda_{0}=1, & N(z)=3.1 \times 10^{-3} h^{-1} n_{-2} R_{10}^{2}\left[(1+z)^{3}-1\right] .
\end{array}
$$

Thus, the $z$-dependence of cumulative number of absorbers is very different in the two cases. 


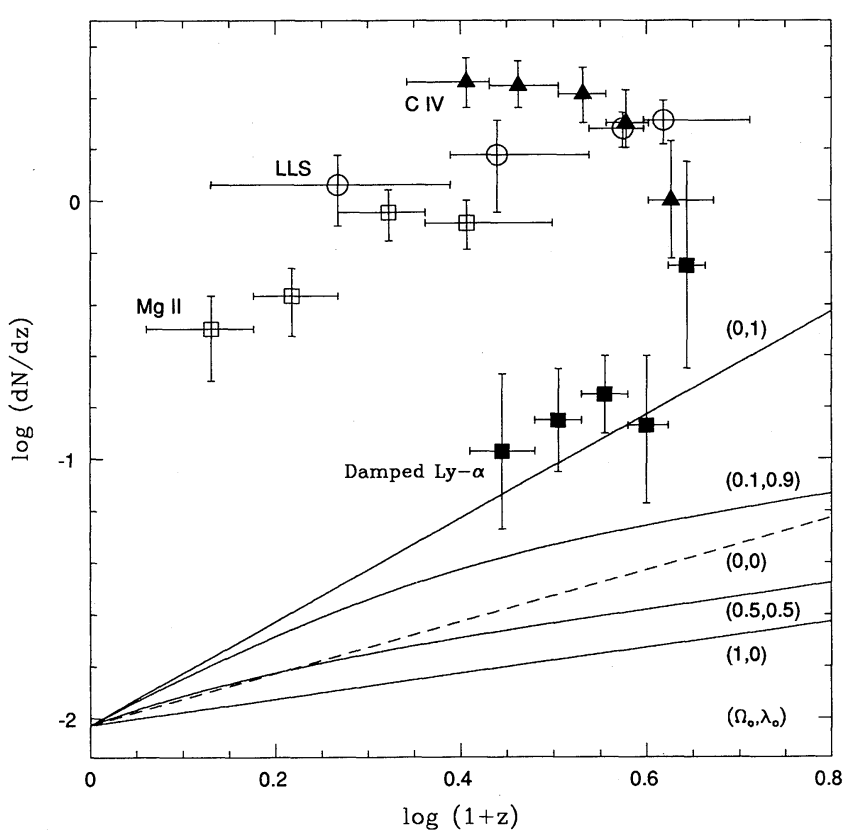

Fig. 1.-Summary of observed number per unit redshift of each absorption line; damped Ly $\alpha, \mathrm{LLS}, \mathrm{Mg}$ II, and $\mathrm{C}$ iv. The expected number per unit redshift in each model universe is also plotted for $\left(\Omega_{0}=1, \lambda_{0}=0\right),(0.5,0.5),(0.1,0.9)$, and $(0,1)$, and $\left(\Omega_{0}=\lambda_{0}=0, k_{0}=-1\right)$ which are normalized at $z=0$ with $R_{10}=n_{-2}=$ $h=1$. Flat models are shown by solid lines, while the open case is a dashed line.

\section{EVOLUTION OF ABSORBERS}

In the previous section, we discussed the expected number of absorbers for the nonevolving case. More generally, we must consider the evolution of absorbers. Evolution might occur in the absorber comoving space density, the size distribution, the abundance of various elements, and the ionization state. Extracting these evolution effects is difficult but necessary to derive any true cosmological effect in the observed number of absorbers.

In each absorption system, the column density (equivalent width) is distributed over a range; the minimum column density is usually limited by the resolution of spectroscopy and the maximum one is determined by the absorber itself. The $\mathrm{H}$ I column density distribution from the Lyman- $\alpha$ forest $\left(N_{\mathrm{HI}}=10^{13}-10^{17} \mathrm{~cm}^{-2}\right)$ and Lyman limit systems $\left(N_{\mathrm{HI}}=10^{17}-10^{20} \mathrm{~cm}^{-2}\right)$ through damped Lyman- $\alpha$ lines $\left(N_{\mathrm{HI}}=10^{19}-10^{22} \mathrm{~cm}^{-2}\right)$ shows a simple power-law form $d n / d N_{\mathrm{HI}} \propto N_{\mathrm{HI}}^{-\beta}(\beta=1.5-1.7)$ (Tytler 1987; Bechtold 1987; Sargent, Steidel, \& Boksenberg 1989). For the origin of this power-law distribution, two possibilities must be considered. One is that the absorbers follow a kind of mass function. The $\mathrm{H}$ I column density depends upon the gas mass in an absorber (Ikeuchi \& Ostriker 1986). The other is that the $\mathrm{H}$ I column density is different according to lines of sight with different impact parameters from the inner to outer part of an absorber (Rees 1988). Here, we take into account these two possibilities, trying to consider an explicit physical model exactly as is done in the usual zero $\Lambda$ analysis for C IV systems (Bergeron \& Ikeuchi 1990). Other interpretations of the column density distribution are clearly possible and might also be considered. Here, however, we just focus on a simple but explicitly physical case in order to contrast the two cosmological models.

\subsection{Column Density Distribution}

We suppose that the space density of absorbers with gas mass $M$ is written as

$$
n_{a}(z, M)=n_{a}\left(0, M_{*}\right)(1+z)^{3}\left(M / M_{*}\right)^{-\delta},
$$

that is, the space density is conserved in comoving coordinate.

By using the impact parameter $l$ of a line of sight from the center of an absorber, the cross section is expressed. Since the column density $\bar{N}$ depends upon the impact parameter, the expected number of spherical absorbers per unit redshift per unit column density is written as

$$
\frac{d^{2} N}{d z d \bar{N}}=\left[\frac{c}{H(z)}\right] \int d M n_{a}(z, M) 2 \pi l(\bar{N}, M)\left|\frac{d l}{d \bar{N}}\right|_{M},
$$

where the impact parameter is supposed to be a function of mass and column density. The integration of mass is chosen appropriately for a given column density. By using equation (2.4) with $k_{0}=0$ and equation (3.1), we rewrite equation (3.2) as

$$
\frac{d^{2} N}{d z d \bar{N}}=\frac{2 \pi c}{H_{0}} \frac{(1+z)^{2} n_{a}\left(0, M_{*}\right)}{\left[\Omega_{0}(1+z)^{3}+\lambda_{0}\right]^{1 / 2}} \int d\left(\frac{M}{M_{*}}\right)\left(\frac{M}{M_{*}}\right)^{-\delta} l(\bar{N}, M)\left|\frac{d l}{d \bar{N}}\right|_{M} .
$$

In the present paper, we assume the gas distribution in an absorber is uniform for simplicity. 
Taking the number density of an ion (atom) within a spherical absorber with radius $R$ as $n_{i}$, the column density at the impact parameter $l$ is given by

$$
\bar{N}_{i}=2 n_{i}\left(R_{a}^{2}-l^{2}\right)^{1 / 2} \sim 2 n_{i} R_{a},
$$

and its derivative becomes

$$
\left|d l / d \bar{N}_{i}\right|=R_{a}\left(R_{a}^{2}-l^{2}\right)^{1 / 2} / \bar{N}_{i} l \simeq R_{a}^{2} / \bar{N}_{i} l
$$

For a spherical absorber, we have

$$
R_{a}=\left(3 M / 4 \pi n_{\mathrm{H}} m\right)^{1 / 3},
$$

where $m$ is the average mass of a particle per hydrogen atom. Summing up these expressions, we get

$$
\frac{d^{2} N}{d z d \bar{N}_{i}}=\frac{2 \pi c}{H_{0}} \frac{(1+z)^{2} n_{a}\left(0, M_{*}\right)}{\left[\Omega_{0}(1+z)^{3}+\lambda_{0}\right]^{1 / 2}}\left(\frac{3 M_{*}}{4 \pi m n_{\mathrm{H}}}\right)^{1 / 3}\left(\frac{M}{M_{*}}\right)^{4 / 3-\delta} \frac{3}{2(4-3 \delta) n_{i}} .
$$

\subsection{Optically Thin H I Systems (Lyman- $\alpha$ Forest, LLS)}

The ionization equilibrium for hydrogen is written as (Black 1981)

$$
n_{\mathrm{HI}} J G_{\mathrm{H}}=n_{\mathrm{H} \text { II }} n_{e} \alpha(T),
$$

where $J$ and $\alpha(T)$ are, respectively, the diffuse UV flux at the Lyman- $\alpha$ limit and the recombination rate. The numerical factor $G_{\mathrm{H}}$ denotes the efficiency of ionization. In the optically thin case, $n_{\mathrm{H} \text { I }} \ll n_{\mathrm{H} \mathrm{II}} \sim n_{e} \sim n_{\mathrm{H}}$ and we get

$$
n_{\mathrm{HI}} \simeq n_{\mathrm{H}}^{2} \alpha(T) / J G_{\mathrm{H}} \text {. }
$$

Then, the $\mathrm{H}$ I column density in equation (3.4) is expressed as

$$
\bar{N}_{\mathrm{HI}} \simeq 2 n_{\mathrm{HI}} R_{a} \simeq \frac{2}{G_{\mathrm{H}}}\left(\frac{3}{4 \pi m}\right)^{1 / 3} n_{\mathrm{H}}^{5 / 3} J^{-1} \alpha M^{1 / 3},
$$

and equation (3.7) becomes

$$
\frac{d^{2} N}{d z d \bar{N}_{\mathrm{HI}}}=\frac{2 \pi c}{H_{0}} \frac{(1+z)^{2} n_{a}\left(0, M_{*}\right)}{\left[\Omega_{0}(1+z)^{3}+\lambda_{0}\right]^{1 / 2}} \frac{3 \times 2^{-5+3 \delta}}{(4-3 \delta)}\left(\frac{4 \pi m}{3 M_{*}}\right)^{1-\delta}\left(\frac{G_{\mathrm{H}}}{\alpha}\right)^{5-3 \delta} n_{\mathrm{H}}^{-9+5 \delta} J^{5-3 \delta} \bar{N}_{\mathrm{H} \mathrm{I}}^{4-3 \delta} .
$$

From the $\mathrm{H}$ I column density distribution of the Lyman- $\alpha$ forest, we can set

$$
\beta=3 \delta-4 \rightarrow \delta=\frac{\beta+4}{3} \text {. }
$$

This means that the $\mathrm{H}$ I column density distribution directly represents the mass function. This simple view probably cannot apply exactly over the entire observed range of column densities but may be useful over the more restricted ranges of interest for particular classes of $\mathrm{H}$ I absorbers.

We assume that the evolution laws of Lyman- $\alpha$ clouds and the diffuse UV flux are simply expressed as

$$
n_{\mathrm{H}}=n_{\mathrm{H}, 0}(1+z)^{x_{1}} \quad \text { and } \quad J=J_{0}(1+z)^{j} .
$$

Assuming the gas temperature to be constant, the number density evolution per unit redshift is given by

$$
\left(\frac{d N}{d z}\right)_{f}=\int_{\bar{N}_{\mathrm{H}, \min }}^{\bar{N}_{\mathrm{H} \mathrm{I}, \max }} d \bar{N}_{\mathrm{HI}} \frac{d^{2} N}{d z d \bar{N}_{\mathrm{HI}}} \propto \frac{(1+z)^{2+x_{1}(5 \delta-9)+j(5-3 \delta)}}{\left[\Omega_{0}(1+z)^{3}+\lambda_{0}\right]^{1 / 2}},
$$

which should be compared with observations. In the latter we assume $j=0$ at $z \geq 2$ and $j=4$ at $z<2$.

In the pressure-confined cloud model for the Lyman- $\alpha$ forest (Ostriker \& Ikeuchi 1983; Ikeuchi \& Ostriker 1986), the cloud temperature is almost constant at $z>2$, and the pressure equilibrium between the cloud and IGM gives

$$
n_{\mathrm{H}}=\frac{P_{I}}{k T} \propto(1+z)^{p} .
$$

We assume the evolution law of IGM pressure to be $P_{I}=P_{I, 0}(1+z)^{p}: p=5$ for the adiabatic expansion case and $p=3.6$ for the shock-heating case (Ikeuchi \& Ostriker 1986). Then, the evolution law of gas density on a cloud in equation (3.13) becomes $x_{1}=p$. The number density evolution in equation (3.14) becomes

$$
\left(\frac{d N}{d z}\right)_{f} \propto \frac{(1+z)^{2+p(5 \beta-7) / 3}}{\left[\Omega_{0}(1+z)^{3}+\lambda_{0}\right]^{1 / 2}} .
$$


TABLE 1

ObServational Data of the Lyman- $\alpha$ Forest $^{a}$

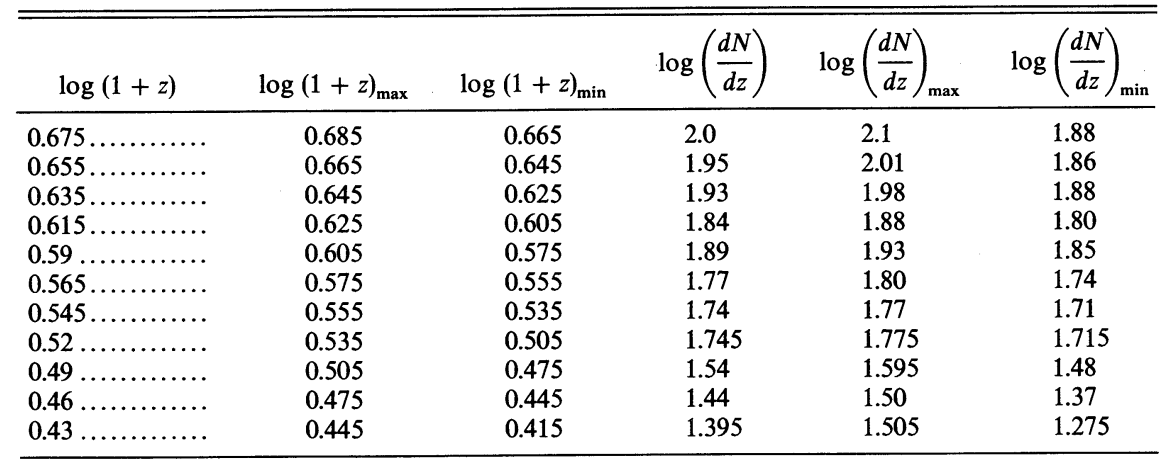

${ }^{a}$ Data are from Fig. 1 of Lu et al. 1991.

In Figure 2, we compare this with observations by Lu, Wolfe, \& Turnshek (1991) for the case of $(p=5, \beta=1.7)$. The data are listed in Table 1.

We see that the observed number density per unit redshift is well reproduced at $z>2$ for the case of $\beta=1.7(\delta=1.9)$ irrespective of the $\lambda_{0}$ value. In contrast, the extrapolation to $z=0$ is quite sensitive to $\lambda_{0}$, but the only available data is for a single quasar (Morris et al. 1991) and is not of sufficient statistical accuracy to be useful in this regard.

For the LLS, the ionization degree of hydrogen is 0.999 even though the $\mathrm{H}$ I column density is $\bar{N}_{\mathrm{HI}} \gtrsim 10^{17.5} \mathrm{~cm}^{-2}$. Therefore, we may consider the LLS as an optically thin system and assume that the LLS is in a gravitational equilibrium regardless of the ambient intergalactic pressure. Then, the number density evolution per unit redshift simply reduces to

$$
\left(\frac{d N}{d z}\right)_{\mathrm{LLS}} \propto \frac{(1+z)^{2}}{\left[\Omega_{0}(1+z)^{3}+\lambda_{0}\right]^{1 / 2}} .
$$

In Figure 3, we illustrate the expected number density evolution per unit redshift as well as the observed one for LLS (Sargent et al. 1989). The data are given in Table 2. The dashed line shows the case of $\Omega_{0}=1$. As is seen, the data at $z<0.5$ may be crucial.

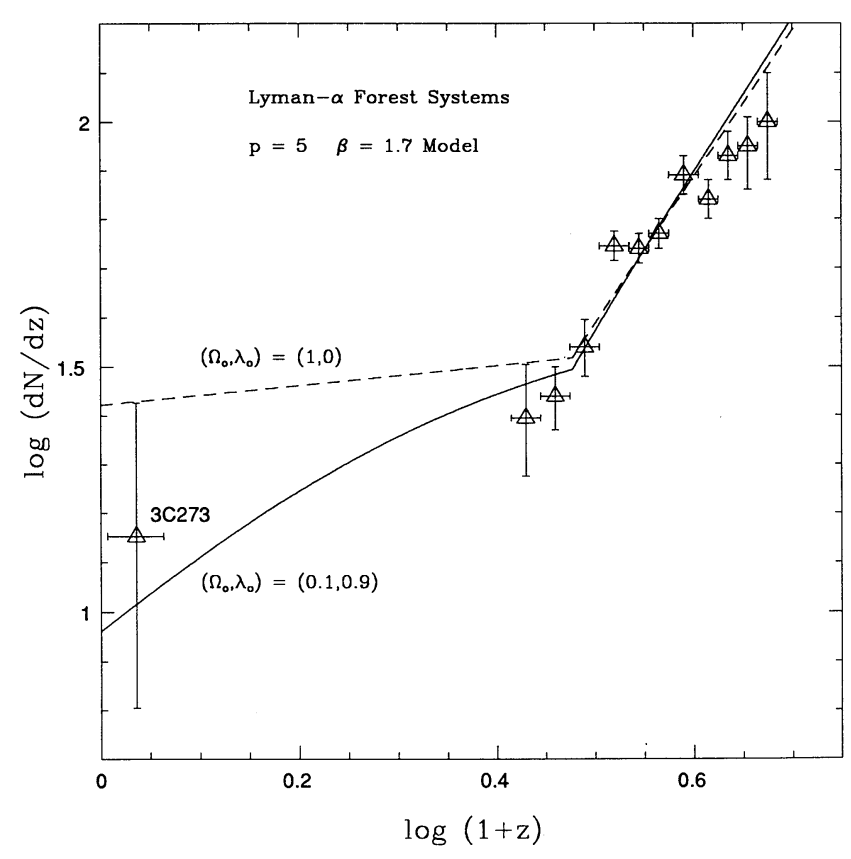

FIG. 2

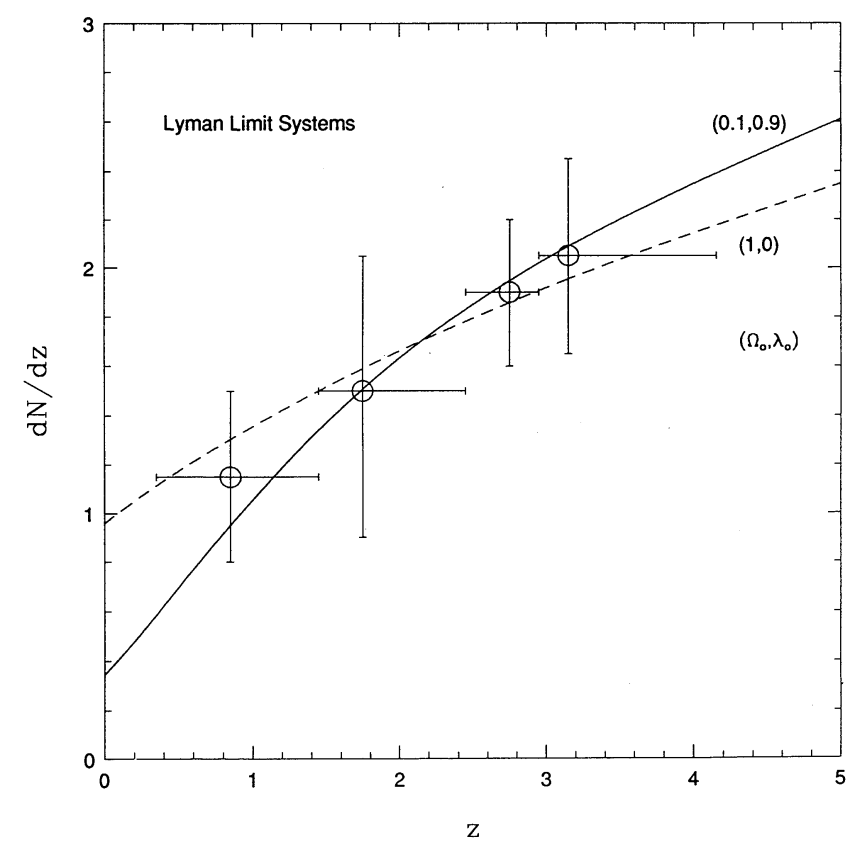

FIG. 3

Fig. 2. - Observed number per unit redshift of the Lyman- $\alpha$ forest (Lu et al. 1991) and predictions by the pressure-confined cloud model. The cases of $\left(\Omega_{0}=0.1\right.$, $\left.\lambda_{0}=0.9\right)$ and $\Omega_{0}=1$ with $(p=5, \beta=1.7)$ are shown for comparison. Observational results of 3C 273 (Morris et al. 1991) are also plotted.

FIG. 3.-Observed number per unit redshift of the LLS (Sargent et al. 1989) as well as the predictions of the models $\left(\Omega_{0}=0.1, \lambda_{0}=0.9\right)$ by a solid line and $\Omega_{0}=1$ by a dashed line. 
TABLE 2

OBSERVATIONAL Data OF LyMAN Limit Systems ${ }^{a}$

\begin{tabular}{cccccc}
\hline \hline$z$ & $z_{\max }$ & $z_{\min }$ & $\left(\frac{d N}{d z}\right)$ & $\left(\frac{d N}{d z}\right)_{\max }$ & $\left(\frac{d N}{d z}\right)_{\min }$ \\
\hline $3.15 \ldots \ldots \ldots$ & 4.15 & 2.95 & 2.05 & 2.45 & 1.65 \\
$2.75 \ldots \ldots \ldots$ & 2.95 & 2.45 & 1.90 & 2.20 & 1.60 \\
$1.75 \ldots \ldots \ldots$ & 2.45 & 1.45 & 1.50 & 2.05 & 0.90 \\
$0.85 \ldots \ldots \ldots$ & 1.45 & 0.35 & 1.15 & 1.50 & 1.40 \\
\hline
\end{tabular}

a Data are from Fig. 5 of Sargent et al. 1989.

\subsection{Optically Thick Systems (Damped Lyman- $\alpha$ Lines)}

As an extreme case, we examine the case of $n_{\mathrm{H}} \sim n_{\mathrm{H}}$ for optically thick, damped Lyman- $\alpha$ systems although this approximation holds only marginally.

The $\mathrm{H}$ I column density is written as

$$
\bar{N}_{\mathrm{HI}} \simeq 2\left(\frac{3}{4 \pi m}\right)^{1 / 3} n_{\mathrm{H}}^{2 / 3} M^{1 / 3}
$$

Substituting this into equation (3.7), we have

$$
\frac{d^{2} N}{d z d \bar{N}_{\mathrm{HI}}}=\frac{2 \pi c}{H_{0}} \frac{(1+z)^{2} n_{a}\left(0, M_{*}\right)}{\left[\Omega_{0}(1+z)^{3}+\lambda_{0}\right]^{1 / 2}}\left(\frac{4 \pi m}{3 M_{*}}\right)^{1-\delta} \frac{3 \times 2^{-5+3 \delta}}{(4-3 \delta)} n_{\mathrm{H}}^{-4+2 \delta} \bar{N}_{\mathrm{H}}^{4-3 \delta} .
$$

Accordingly, we also get the same index for $\mathrm{H}$ I column density distribution as for the Lyman- $\alpha$ forest except that the normalization is different.

If both the optically thin and thick systems follow the same mass function, the $\mathrm{H}$ I column density distributions would have the same index $\beta$ but different normalization as

$$
R \equiv\left(\frac{d^{2} N}{d z d \bar{N}_{\mathrm{HI}}}\right)_{\text {thin }} /\left(\frac{d^{2} N}{d z d \bar{N}_{\mathrm{HI}}}\right)_{\text {thick }}=\left(\frac{\alpha n_{\mathrm{H}, f}^{2}}{J G_{\mathrm{H}}}\right)^{(\beta-1)} \frac{n_{\mathrm{H}, f}^{-(\beta+1) / 3}}{n_{\mathrm{H}, d}^{(2 \beta-4) / 3}},
$$

where $n_{\mathrm{H}, f}$ and $n_{\mathrm{H}, d}$ are, respectively, the gas density of an optically thin and thick system. From equation (3.8), this ratio becomes

$$
R=\left(n_{\mathrm{HI}, f} / n_{\mathrm{H}, d}\right)^{\beta-1}\left(n_{\mathrm{H}, f} / n_{\mathrm{H}, d}\right)^{-(\beta+1) / 3} .
$$

In the minihalo model, the optically thin and thick systems correspond to the lines of sight at the outer and inner region, respectively, of a single minihalo. For an example we get $n_{\mathrm{H}, f} \simeq 10^{-7} \mathrm{~cm}^{-3}, n_{\mathrm{H}, f} \simeq 10^{-4} \mathrm{~cm}^{-3}$, and $n_{\mathrm{H}, d} \simeq 10^{-2} \mathrm{~cm}{ }^{-3}(\mathrm{Ikeuchi}$, Murakami, \& Rees 1988), which gives

$$
R=10^{-5(\beta-1)} 10^{2(\beta+1) / 3} \simeq 10^{-1.7} .
$$

This means that the optically thin and thick systems show the same power index for column density distribution but thick systems are more numerous by a factor of $R$.

For these optically thick systems, we assume the evolution law of absorbers to be $n_{\mathrm{H}}=n_{\mathrm{H}, 0}(1+z)^{x_{2}}$, and the number density evolution per unit redshift becomes

$$
\left(\frac{d N}{d z}\right)_{d} \propto \frac{(1+z)^{2+x_{2}(2 \delta-4)}}{\left[\Omega_{0}(1+z)^{3}+\lambda_{0}\right]^{1 / 2}} .
$$

Since these optically thick systems are already settled to a gravitational equilibrium state and $x_{2}=0$, we have

$$
\left(\frac{d N}{d z}\right)_{d} \propto \frac{(1+z)^{2}}{\left[\Omega_{0}(1+z)^{3}+\lambda_{0}\right]^{1 / 2}} .
$$

In Figure 4, we illustrate the expected number density evolution per unit redshift as well as the observed one for the damped Lyman- $\alpha$ systems (Lanzetta et al. 1991). The data are given in Table 3. The dashed lines show the case of $\Omega_{0}=1$. The difference between the cases of $\left(\Omega_{0}=0.1, \lambda_{0}=0.9\right)$ and $\Omega_{0}=1$ are small since the observations are limited to $z>z_{c}$. However, at $z<z_{c}$ the predictions of these two cases differ by more than a factor of 2 . The nonzero $\Lambda$ case predicts a smaller number at the epoch $z<0.5$.

\subsection{Metallic Line Systems}

Since the apparent evolution of metallic line systems such as C IV, Si IV, and Mg II may be a curve of growth effect (Tytler 1987) and depends upon the cutoff equivalent widths of samples, it may be dangerous to reach a conclusion by using the $d n / d z$ results. However, it may still be valuable to present a simple analysis in the nonzero $\Lambda$ universe in comparison with the usual zero- $\Lambda$ one. 
No. 2, 1992

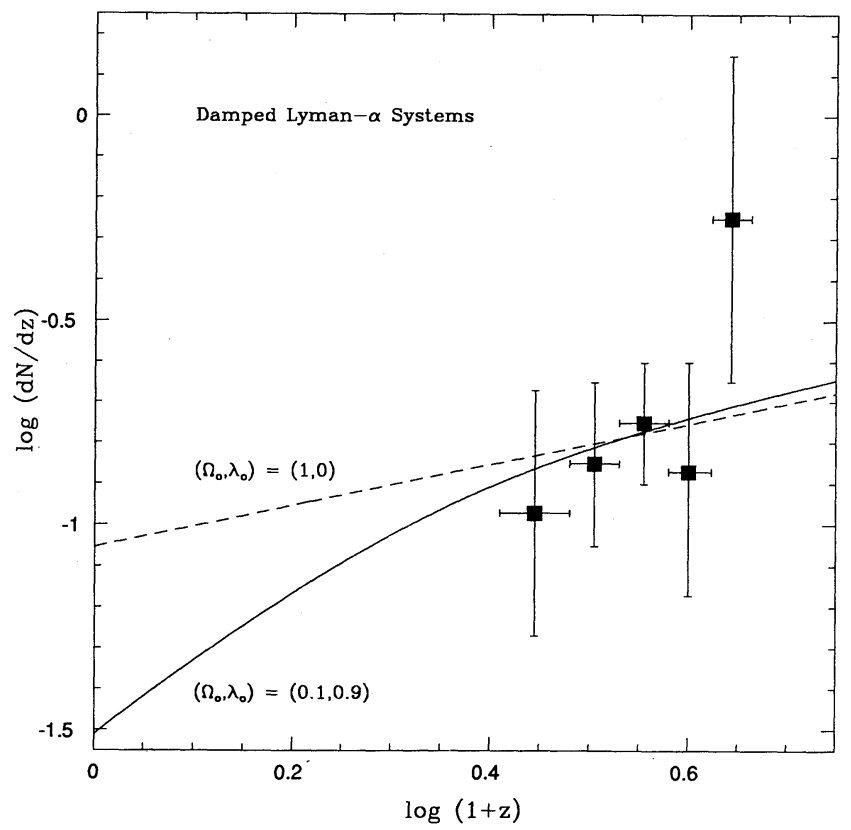

Fig. 4.-The same as in Fig. 3, but for the damped Ly $\alpha$ line (Lanzetta et al. 1991)

The column density of metallic ion $x$ is related to the total hydrogen column density according to

$$
\bar{N}_{x}=\bar{N}_{\mathrm{H}}\left(\frac{n_{x}}{n_{\mathrm{H}}}\right) i(x),
$$

where $n_{x}$ and $i(x)$ are the abundance of an atomic species $x$ and its ionization stage. For the photoionization model, the ionization state is determined only by the ionization parameter $U$ with a simple dependence to be

$$
i(x)=\left(U / U_{0}\right)^{\eta} .
$$

Then, the H I column density distribution in equation (3.19) is converted to the column density distribution of $x$-ion by

$$
\frac{d^{2} N}{d z d \bar{N}_{x}}=\frac{2 \pi c}{H_{0}} \frac{(1+z)^{2} n_{a}\left(0, M_{*}\right)}{\left[\Omega_{0}(1+z)^{3}+\lambda_{0}\right]^{1 / 2}}\left(\frac{4 \pi m}{3 M_{*}}\right)^{1-\delta} \frac{3 \times 2^{-5+3 \delta}}{(4-3 \delta)} n_{\mathrm{H}}^{-4+2 \delta} Z_{x}^{-5+3 \delta}\left(\frac{U}{U_{0}}\right)^{-\eta(5-3 \delta)} \bar{N}_{x}^{4-3 \delta},
$$

where we set $Z_{x} \equiv n_{x} / n_{\mathrm{H}}$. Assuming the evolution laws to be

$$
n_{\mathrm{H}}=n_{\mathrm{H}, 0}(1+z)^{x_{3}}, \quad Z_{x}=Z_{x, 0}(1+z)^{v}, \quad \text { and } \quad J=J_{0}(1+z)^{j},
$$

we have

$$
\left(\frac{d N}{d z}\right)_{m} \propto \frac{(1+z)^{2+x_{3}[-4+2 \delta+(5-3 \delta) \eta]+v(-5+3 \delta)+j(-5+3 \delta) \eta}}{\left[\Omega_{0}(1+z)^{3}+\lambda_{0}\right]^{1 / 2}},
$$

where we use the relation

$$
U \propto\left(J / n_{\mathrm{H}}\right) \propto(1+z)^{j-x_{3}} .
$$

TABLE 3

Observational Data of Damped Lyman- $\alpha$ Lines $^{a}$

\begin{tabular}{cccccc}
\hline \hline $\log (1+z)$ & $\log (1+z)_{\max }$ & $\log (1+z)_{\min }$ & $\log \left(\frac{d N}{d z}\right)$ & $\log \left(\frac{d N}{d z}\right)_{\max }$ & $\log \left(\frac{d N}{d z}\right)_{\min }$ \\
\hline $0.643 \ldots \ldots \ldots$ & 0.663 & 0.623 & -0.25 & 0.15 & -0.65 \\
$0.600 \ldots \ldots \ldots$ & 0.623 & 0.580 & -0.87 & -0.60 & -1.17 \\
$0.555 \ldots \ldots \ldots$ & 0.580 & 0.530 & -0.75 & -0.60 & -0.90 \\
$0.505 \ldots \ldots \ldots$ & 0.530 & 0.480 & -0.85 & -0.65 & -1.05 \\
$0.445 \ldots \ldots \ldots$ & 0.480 & 0.410 & -0.97 & -0.67 & -1.27 \\
\hline
\end{tabular}

a Data are from Fig. 8 of Lanzetta et al. 1991. 
TABLE 4

Observational Data of C IV Lines ${ }^{a}$

\begin{tabular}{cccccc}
\hline \hline$z$ & $z_{\max }$ & $z_{\min }$ & $\left(\frac{d N}{d z}\right)$ & $\left(\frac{d N}{d z}\right)_{\max }$ & $\left(\frac{d N}{d z}\right)_{\min }$ \\
\hline $3.23 \ldots \ldots \ldots$ & 3.70 & 3.00 & 1.00 & 1.70 & 0.60 \\
$2.78 \ldots \ldots \ldots$. & 3.00 & 2.60 & 2.00 & 2.70 & 1.60 \\
$2.40 \ldots \ldots \ldots$. & 2.60 & 2.20 & 2.60 & 3.30 & 2.00 \\
$1.90 \ldots \ldots \ldots$. & 2.20 & 1.70 & 2.80 & 3.50 & 2.30 \\
$1.55 \ldots \ldots \ldots$. & 1.70 & 1.20 & 2.90 & 3.60 & 2.30 \\
\hline
\end{tabular}

${ }^{a}$ Data are from Fig. 3 of Steidel 1990.

As in Bergeron \& Ikeuchi (1990), the column density ratio of the Si IV and C IV ions shows a simple evolution law

$$
\bar{N}_{\mathrm{Si} \mathrm{IV}} / \bar{N}_{\mathrm{CIV}} \propto(1+z)^{s}, \quad S \simeq 3.0 .
$$

In the photoionization model, this ratio depends only upon the ionization parameter, if the absorptions occur in the same region,

$$
\bar{N}_{\mathrm{Si} \mathrm{IV}} / \bar{N}_{\mathrm{CIV}} \propto U^{-C} \propto\left(J / n_{\mathrm{H}}\right)^{-C} \propto(1+z)^{\left(x_{3}-j\right) c},
$$

where we assume the abundance ratio of silicon to carbon to be constant in any epoch. According to the photoionization model by Bergeron \& Stasinska (1986), the power index is $C \simeq 1.2$ (see, also, Bergeron \& Ikeuchi 1990). Then, we get the evolution law at $z>2$ (i.e., $j=0$ ) for the gas density of an absorber as

$$
x_{3}=S / C \sim 2.5 .
$$

At the $\mathrm{H}$ I column density of $\mathrm{C}$ IV absorbing systems, $10^{15.5}-10^{21.5} \mathrm{~cm}^{-2}$, the power index of $\mathrm{H}$ I column density distribution is between $\beta \simeq 0.8$ and 1.5 (Bechtold 1987). Here, we take $\beta \simeq 1.0$, i.e., $\delta=5 / 3$. Then, we have the number density evolution per unit redshift in equation (3.29)

$$
\left(\frac{d N}{d z}\right)_{\mathrm{C} \mathrm{IV}} \propto \frac{(1+z)^{1 / 3}}{\left[\Omega_{0}(1+z)^{3}+\lambda_{0}\right]^{1 / 2}} .
$$

In Figure 5, we illustrate this evolution law as well as the observations (Steidel 1990), with the data listed in Table 4.

The observed number density evolution per unit redshift of $\mathrm{C}$ IV systems is well fitted by this model at $z>1.5$. The difference between the cases of $\Omega_{0}=1$ and $\left(\Omega_{0}=0.1, \lambda_{0}=0.9\right)$ appears clearly at $z \lesssim 1$. The nonzero $\Lambda$ universe predicts a smaller expected number of $\mathrm{C}$ IV systems at $z \lesssim 1$.

For the $\mathrm{Mg}$ II systems, we take $\beta \simeq 1.0(\delta=5 / 3)$ because $N_{\mathrm{HI}} \simeq 10^{18} \mathrm{~cm}^{-2}$ in them. The expected number density evolution per unit redshift becomes

$$
\left(\frac{d N}{d z}\right)_{\mathrm{Mg} \mathrm{II}} \propto \frac{(1+z)^{2-2 x_{3} / 3}}{\left[\Omega_{0}(1+z)^{3}+\lambda_{0}\right]^{1 / 2}} .
$$

Since $\mathrm{Mg}$ II systems are observed around $z_{c} \sim 1$, the effect of nonzero $\Lambda$ appears clearly. In Figure 6 we illustrate the observed number density per unit redshift (Sargent, Steidel, \& Boksenberg 1988b) as well as those for the cases of $\left(\lambda_{0}=0.1, \Omega_{0}=0.9\right)$ and $\Omega_{0}=1$ with $x_{3}=0$ (nonevolving absorbers). The data are in Table 5. The observed number evolution is not reproduced by the $\Omega_{0}=1$ case. Nonzero $\Lambda$ models marginally reproduce the observational tendency: slow decrease at $z>1$ and steep decrease at $z<1$. However, the published samples are sensitive only to doublets stronger than $0.6 \AA$, and the equivalent width distribution function shows no sign of a cutoff near the above value. Moreover, there is a trend for doublets to become weaker at lower redshifts. This may indicate the evolution of $\mathrm{Mg}$ II absorbers is contrary to the assumption $x_{3}=0$.

\section{5. $\mathrm{H}$ I Absorption by Intergalactic Gas}

The $\mathrm{H}$ I absorption by pervasive intergalactic gas is well known as the Gunn-Peterson effect. The optical depth of $\mathrm{H}$ I gas at redshift $z$ is given by

$$
\tau_{\mathrm{HI}}(z)=n_{\mathrm{HI}} \sigma_{\mathrm{HI}} \frac{c}{H(z)}=4.1 \times 10^{10} n_{\mathrm{HI}} h^{-1}\left[\Omega_{0}(1+z)^{3}+\lambda_{0}\right]^{-1 / 2},
$$

where $\sigma_{\mathrm{HI}}$ is the absorption cross section of $\mathrm{UV}$ photons by $\mathrm{H}$ I gas, $\sigma_{\mathrm{HI}}=4.5 \times 10^{-18} \mathrm{~cm}^{2}$. At present, only upper limits for $\mathrm{H} \mathrm{I}$ optical depth have been reported. This means the intergalactic gas is very optically thin. Assuming the ionization equilibrium by diffuse UV flux as in equation (3.9), the optical depth is rewritten as

$$
\tau_{\mathrm{HI}}(z) \simeq n_{I}^{2} \sigma_{\mathrm{HI}} \frac{c}{H(z)} \frac{\alpha\left(T_{I}\right)}{J G_{\mathrm{H}}},
$$




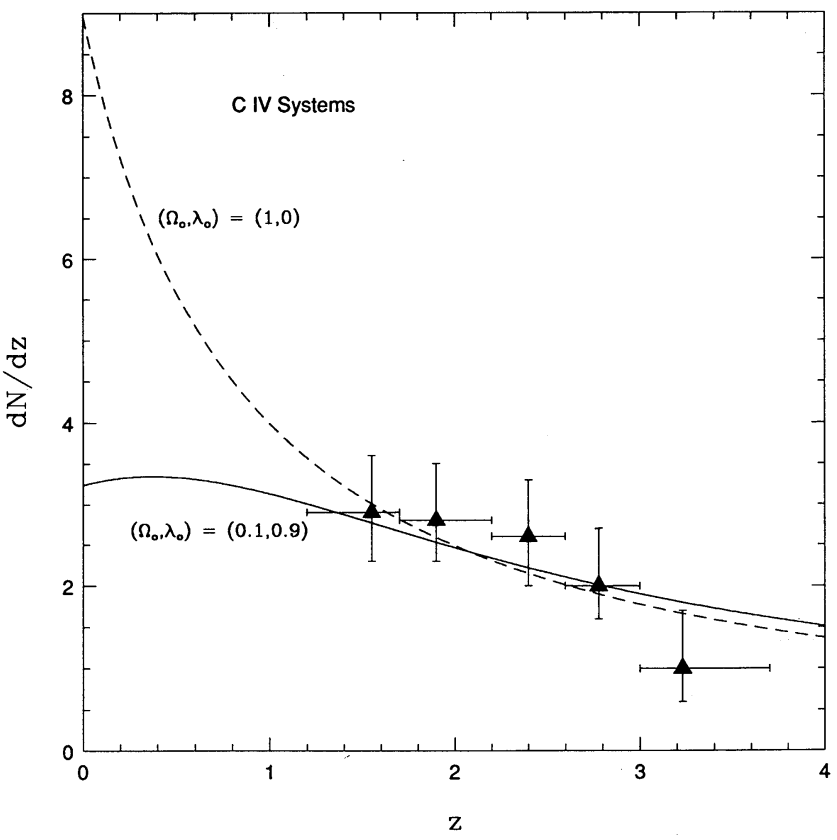

FIG. 5

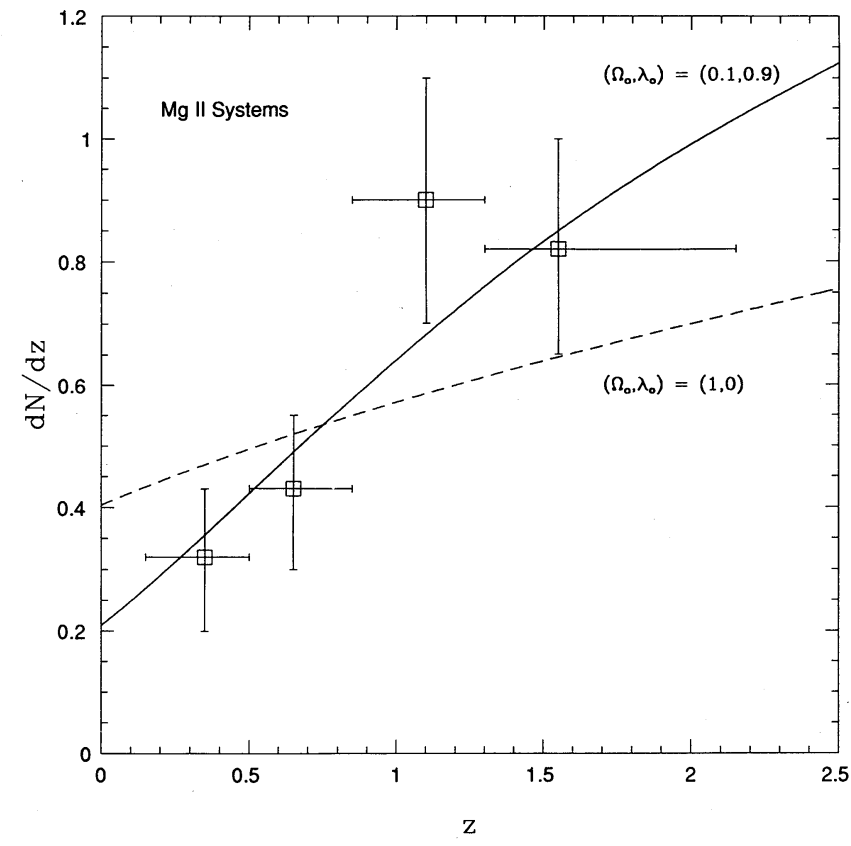

FIG. 6

Fig. 5.-The same as in Fig. 3, but for the C Iv lines (Steidel 1990)

Fig. 6.-The same as in Fig. 3, but for the Mg II lines (Sargent et al. 1988b)

where the suffix $I$ denotes the quantities of intergalactic gas. The recombination rate is approximated around $T_{I} \sim 10^{4} \mathrm{~K}$ by $(\mathrm{Black}$ 1981)

$$
\alpha\left(T_{I}\right)=\alpha_{0} T_{I}^{-3 / 4} \simeq 4.4 \times 10^{-10} T_{I}^{-3 / 4} .
$$

By using the pressure, equation (3.37) is expressed by

$$
\tau_{\mathrm{H} \mathrm{I}}(z) \simeq P_{I}^{-3 / 4} n_{I}^{11 / 4} \frac{\sigma_{\mathrm{HI}} c \alpha_{0} k^{3 / 4}}{H(z) J G_{\mathrm{H}}},
$$

where $k$ is the Boltzmann constant.

The evolution laws of intergalactic gas and diffuse UV flux are taken to be

$$
P_{I}=P_{I, 0}(1+z)^{p}, \quad n_{I}=n_{I, 0}(1+z)^{3}, \quad \text { and } \quad J=J_{0}(1+z)^{j},
$$

where we assume the comoving gas density is conserved. For the adiabatic expansion case, $p=5$ and for the shock heating case, $p=3.6$. Applying equation (3.40) to equation (3.39), the exact expression for the optical depth becomes

$$
\tau_{\mathrm{HI}}(z) \simeq \frac{\sigma_{\mathrm{HI}} c \alpha_{0} k^{3 / 4}}{J_{0} G_{\mathrm{H}}} n_{\text {crit, } 0}^{11 / 4} P_{I, 0}^{-3 / 4} \frac{\Omega_{I, 0}^{11 / 4}(1+z)^{(-3 p+33) / 4-j}}{H_{0}\left[\Omega_{0}(1+z)^{3}+\lambda_{0}\right]^{1 / 2}},
$$

where $n_{\text {crit }, 0}=3 H_{0}^{2} / 8 \pi G m \simeq 10^{-5} h^{2} \mathrm{~cm}^{-3}$ and $\Omega_{I, 0}=n_{I, 0} / n_{\text {crit }, 0}$.

From equation (3.41), we can see that if the observed (upper) limits of $\mathbf{H}_{\mathrm{I}}$ optical depth at a high redshift (say $z=2.64$ ) and a low redshift (say $z=0.0$ ) are the same, the upper limit for $P_{I, 0}^{-3 / 4} \Omega_{I, 0}^{11 / 4}$ is set from the result of the higher redshift as long as the power index of $(1+z)$ is positive, i.e., $(33-3 p) / 4-j-3 / 2 \gtrsim 0$.

The most severe observed upper limit on the $\mathrm{H}$ I optical depth due to the Gunn-Peterson test is given by Steidel \& Sargent (1987) as $\tau_{\mathrm{HI}}<0.02( \pm 0.03)$ at $\langle z\rangle=2.64$. The predicted $\mathrm{H}_{\mathrm{I}}$ optical depth in equation (3.41) must run below this upper limit.

TABLE 5

ObSERvational Data of Mg in Lines ${ }^{\mathrm{a}}$

\begin{tabular}{cccccc}
\hline \hline$z$ & $z_{\max }$ & $z_{\min }$ & $\left(\frac{d N}{d z}\right)$ & $\left(\frac{d N}{d z}\right)_{\max }$ & $\left(\frac{d N}{d z}\right)_{\min }$ \\
\hline $1.55 \ldots \ldots \ldots$ & 2.15 & 1.30 & 0.82 & 1.00 & 0.65 \\
$1.10 \ldots \ldots \ldots$ & 1.30 & 0.85 & 0.90 & 1.10 & 0.70 \\
$0.65 \ldots \ldots \ldots$ & 0.85 & 0.50 & 0.43 & 0.55 & 0.30 \\
$0.35 \ldots \ldots \ldots$ & 0.50 & 0.15 & 0.32 & 0.43 & 0.20 \\
\hline
\end{tabular}

${ }^{\text {a }}$ Data are from Fig. 7 of Sargent et al. 1988b. 
From this condition we can determine the allowed range for the present pressure and density of IGM for $p=5, h=1$, and $J_{0}(z=0)$ $=10^{-21} / 3^{4}$ as

$$
\begin{aligned}
\tilde{P}_{I, 0}^{-3 / 4} \Omega_{I, 0}^{11 / 4} & <2.9 \times 10^{-6} & & \left(\Omega_{0}=1\right), \\
& <9.8 \times 10^{-7} & & \left(\Omega_{0}=0.1, \lambda_{0}=0.9\right),
\end{aligned}
$$

where we put $\tilde{P}_{I}=P_{I} / k$. The upper limit $\tau_{\mathrm{HI}}<0.04$ at $z=0.158$ of $3 \mathrm{C} 273$ gives

$$
\begin{aligned}
\tilde{P}_{I, 0}^{-3 / 4} \Omega_{I, 0}^{11 / 4} & <3.2 \times 10^{-4} & & \left(\Omega_{0}=1\right), \\
& <2.6 \times 10^{-4} & & \left(\Omega_{0}=0.1, \lambda_{0}=0.9\right),
\end{aligned}
$$

In Figure 7, we illustrate the allowed range for $\tilde{P}_{I, 0}$ and $\Omega_{I, 0}$.

\subsection{Thomson Scattering and y-Parameter}

The optical depth of Thomson scattering of CBR photons by electrons in the ionized IGM is given by

$$
\tau_{\mathrm{T}}=\int_{0}^{z} \sigma_{\mathrm{T}} n_{I, e}(z) \frac{c d z}{H(z)(1+z)},
$$

where $\sigma_{\mathrm{T}}$ is the cross section of Thomson scattering, $\sigma_{\mathrm{T}}=6.6 \times 10^{-25} \mathrm{~cm}^{2}$. The corresponding $y$-parameter of inverse Compton scattering is

$$
y=\int_{0}^{z} \frac{k T_{I, e}(z)}{m_{e} c^{2}} \sigma_{\mathrm{T}} n_{I, e}(z) \frac{c d z}{H(z)(1+z)} .
$$

[Note that the $(1+z)$ factor was omitted from the corresponding eqs. (17) and (18) of Ikeuchi \& Turner 1991. As a consequence the numerical results given in that paper's eq. (20) along with the corresponding lines in its Fig. 4 are too small by a factor of just about 4.] For an almost wholly ionized IGM, $n_{e} \sim n_{I}$, equation (3.43) is rewritten as

$$
\tau_{\mathrm{T}}=\frac{\sigma_{\mathrm{T}} c n_{I, 0}}{H_{0}} \int_{0}^{z} \frac{(1+z)^{2}}{\left[\Omega_{0}(1+z)^{3}+\lambda_{0}\right]^{1 / 2}} d z,
$$

and the $y$-parameter becomes

$$
y=\frac{\sigma_{\mathrm{T}} c P_{I, 0}}{2 m_{e} c^{2} H_{0}} \int_{0}^{z} \frac{(1+z)^{p-1}}{\left[\Omega_{0}(1+z)^{3}+\lambda_{0}\right]^{1 / 2}} d z .
$$

The Compton $y$-parameter is limited to $y<10^{-3}$ by $C O B E$ (Mather et al. 1990).

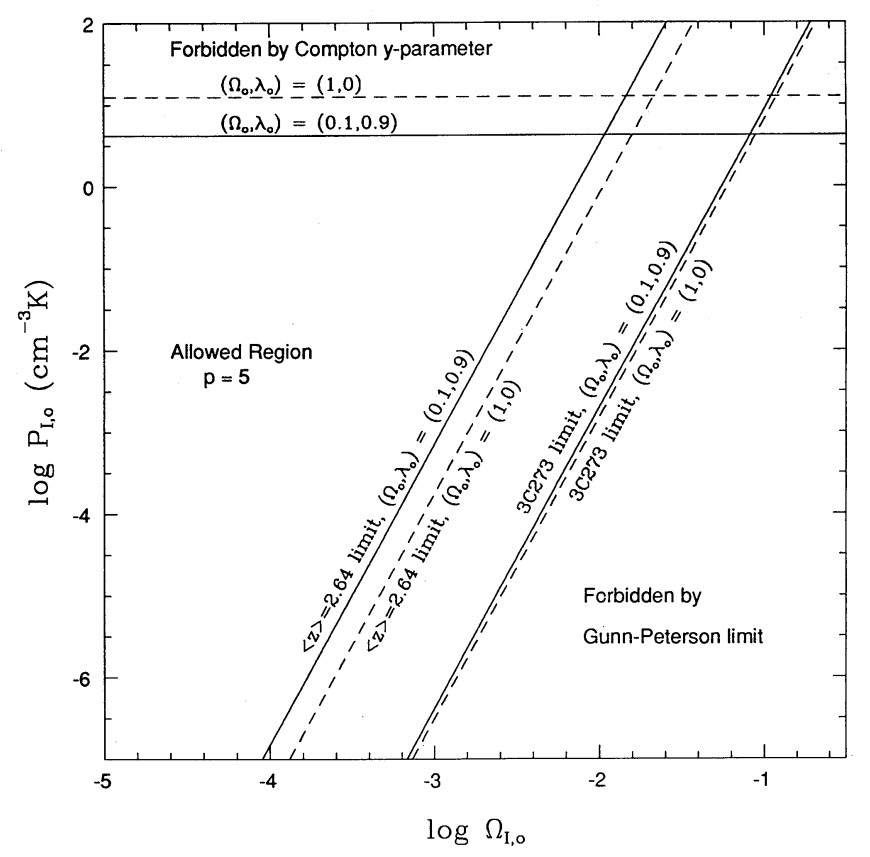

FIG. 7.-The allowed range of the pressure and mean number density parameter of the IGM determined by the G-P test at $\langle z\rangle=2.64$ and $z=0.158(3 \mathrm{C} 273)$, and by the $y$-parameter for $p=5$ and $z_{i}=5$. 
This gives an upper limit to the pressure for a given redshift corresponding to the ionization epoch of IGM

$$
\tilde{P}_{I, 0}<1.9 \times 10^{3} h / F(z, p)
$$

with

$$
F(z, p)=\int_{0}^{z_{i}} \frac{(1+z)^{p-1}}{\left[\Omega_{0}(1+z)^{3}+\lambda_{0}\right]^{1 / 2}} d z .
$$

For $p=5$, we get the upper limit of IGM pressure as

$$
\begin{aligned}
\tilde{P}_{I, 0} & <12.6 h\left(\Omega_{0}=1\right), \\
& <4.2 h\left(\Omega_{0}=0.1, \lambda_{0}=0.9\right) .
\end{aligned}
$$

In Figure 7, we also plot the upper limits of present IGM pressure in equation (3.49) for $p=5$ with $\left(\Omega_{0}=0.1, \lambda_{0}=0.9\right)$ and $\Omega_{0}=1$ in the case of $z_{i}=5$.

Combining the Gunn-Peterson test and this $y$-parameter limit, we can get a little more severe constraints on the IGM for the nonzero A model than the zero $\Lambda$ model.

\subsection{Dust Absorption}

Taking the number density per unit redshift of absorbers in which dust is distributed to be $(d N / d z)_{D}$, the optical depth at frequency $v$ may be written as

$$
\tau_{D, v}=\int_{0}^{z}\left(\frac{d N}{d z}\right)_{D} \kappa_{v}(z) d z,
$$

where $\kappa_{v}(z)$ is the dust opacity at the frequency $v$. As indicated by Fall, Pei, \& McMahon (1989), the damped Lyman- $\alpha$ line systems may contribute to the dust absorption with different absorption laws than in our Galaxy. In general, the expected high- $z$ effects will increase as $\Omega_{0}^{-1 / 2}=\left(1-\lambda_{0}\right)^{-1 / 2}$.

\section{DISCUSSION AND PREDICTIONS}

As expected, the difference in absorption systems of QSOs between zero $\Lambda$ and nonzero $\Lambda$ is strongest at the epoch $z<z_{c} \sim 1$. Therefore, the observations at the wavelength ranges, $\lambda=\left[\lambda_{r}, \lambda_{r}\left(1+z_{c}\right)\right]$, are crucial, $\lambda_{r}$ being the rest frame wavelength of absorbing ions. Representative wavelengths are $\lambda_{r}=912 \AA$ (Lyman limit of hydrogen), $\lambda_{r}=1216 \AA$ (Ly $\left.\alpha\right), \lambda_{r}=1548,1550 \AA$ (C IV), and $\lambda_{r}=2796,2804 \AA\left(\mathrm{Mg}\right.$ II). Other lines such as $\lambda_{r}=1032,1038 \AA(\mathrm{O} \mathrm{vI}), \lambda_{r}=1259,1243 \AA\left(\mathrm{N}\right.$ v), $\lambda_{r}=1145 \AA\left(\mathrm{Fe}\right.$ II), $\lambda_{0}=1190$, $1193 \AA$ (Si II) and $\lambda_{r}=1394,1403 \AA(\mathrm{Si}$ IV) also may be important as subsidary lines. Since almost all of these lines are in the UV range for $z_{c} \sim 1$, high-resolution spectroscopy by $H S T$ and $A S T R O$ should be very informative.

Generally speaking, the expected number of absorption systems in the nonzero $\Lambda$ universe is smaller at $z<z_{c}$ than that in the $\Omega_{0}=1$ universe if we fit the model calculations to the observations at $z \sim 2$. That is, a large $\Lambda$ produces a rapid decrease of absorbers even if the absorbers themselves do not evolve much. Therefore, surveys of absorption spectra of QSOs with redshifts less than $z_{c} \sim 1$ will allow the most important test.

Table 6 gives predicted values of $d n / d z$ at $z=0$ for our two reference cosmologies for each type of absorption discussed in the preceding section. These are based on formal least-squares fits to the data listed in the other tables; $\pm 1 \sigma$ ranges are also indicated.

The Gunn-Peterson test in nearby QSOs is also valuable for probing the IGM and $\Lambda$ term. As shown in Figure 8, the $\mathrm{H}$ I optical depth in equation (3.41) shows remarkably different behaviors for the two cases of $\Omega_{0}=1$ and $\left(\Omega_{0}=0.1, \lambda_{0}=0.9\right)$ at $z \leq 2$, at which epoch we assume $j=4$. Then the low- $z$ Gunn-Peterson test using $H S T$ is crucial for probing the IGM and the $\Lambda$ term.

The detection probability of damped Lyman- $\alpha$ lines in nearby QSOs would also give important information on the origin of

\begin{tabular}{|c|c|c|c|c|c|}
\hline \multirow[b]{2}{*}{ ABSORPTION TYPE } & \multicolumn{2}{|c|}{$\left(\Omega_{0}, \lambda_{0}\right)=(1,0)$} & \multicolumn{2}{|c|}{$\left(\Omega_{0}, \lambda_{0}\right)=(0.1,0.9)$} & \multirow{2}{*}{$\begin{array}{l}\text { FitTING } \\
\text { EQUATION }\end{array}$} \\
\hline & Best Fit & $\pm 1 \sigma$ & Best Fit & $\pm 1 \sigma$ & \\
\hline \multicolumn{6}{|l|}{ Lyman- $\alpha$ forest } \\
\hline$W_{r} \geq 360 \mathrm{~m} \AA$ & 26.4 & $25.6-27.3$ & 9.15 & $8.85-9.45$ & $\begin{array}{c}(3.16) \\
p=5, \beta=1.7\end{array}$ \\
\hline Lyman limit & 0.959 & $0.854-1.06$ & 0.344 & $0.307-0.381$ & (3.17) \\
\hline Damped Lyman- $\alpha \ldots$ & 0.088 & $0.070-0.11$ & 0.031 & $0.024-0.039$ & (3.24) \\
\hline C IV $1549 \AA$. & 8.45 & $7.91-10.0$ & 3.24 & $2.85-3.65$ & (3.34) \\
\hline \multirow[t]{2}{*}{ Mg II $2800 \AA \ldots \ldots \ldots . .}$. & $0.404^{\mathrm{a}}$ & $0.350-0.458$ & 0.209 & $0.182-0.236$ & (3.35) \\
\hline & & & & & $x_{3}=0$ \\
\hline
\end{tabular}
absorbers. As shown in Figure 1, the observed number is better reproduced by a $\lambda_{0}=0.9, \Omega_{0}=0.1$ model than by $\lambda_{0}=0$ models if the absorbers are normal galaxies of the same space density as nearby galaxies.

TABLE 6

LEAST-SQUARES NoRmalizations of $d N / d z$ Fits $(d N / d z)$ at $z=0$

a Poor fit. 


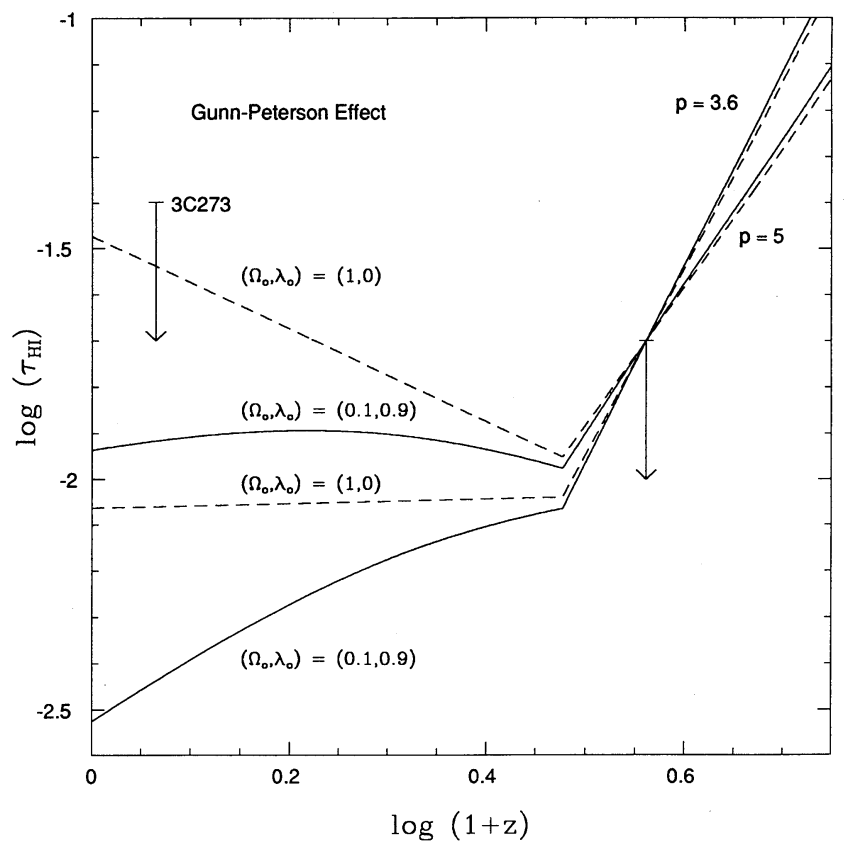

FIG. 8. -The upper limit of the H I optical depth of IGM. The predictions for the case of $\left(\Omega_{0}=0.1, \lambda_{0}=0.9\right)$ and $\Omega_{0}=1$ are normalized to be $\tau_{\mathrm{HI}}=0.02$ at $z=2.64$. The $H S T$ result for $3 \mathrm{C} 273$ (Bahcall et al. 1991) is also plotted.

For Mg II systems, more accurate data at $z<0.5$ and at $z>2$ are desirable. The steep decrease at $z<0.5$ is suggestive of a nonzero $\Lambda$ model, and this should be studied further. At $z>2$, the nonzero $\Lambda$ model predicts much more Mg il absorption systems than the $\Omega_{0}=1$ model. This observation should be possible soon using ground-based telescopes in the near infrared band $(\lambda>9000 \AA)$.

Given that more definite conclusions must await the eminent extension of detailed IGM absorption observations to $z \lesssim 1$, what may be said at this point on the basis of available data? The two salient points are as follows:

1. The frequency of high column density $\mathrm{H}$ I and metal absorption lines are closer to those expected from the properties of present galaxies for $\Lambda$-dominated cosmologies; however, there is still a significant discrepancy for all lines.

2. A relatively high frequency of low- $z$ absorption lines, as suggested by some early $H S T$ and $A S T R O$ results, would be most easily understood in a $\Lambda=0$ cosmology.

We thank H. Suzuki-san for her careful wordprocessing. J. P. Ostriker, J. Miralda-Escude, and O. Lahav provided useful comments on an earlier version of this paper. We are also pleased to receive valuable comments from an anonymous referee. This research was performed with the support of the Scientific Aid of the Ministry of Science, Culture and Education of Japan (C0340252), and from NASA grant NAGW-2448. We also acknowledge the hospitality of the Aspen Center for Physics.

\section{REFERENCES}

Bahcall, J. N., et al. 1991, ApJ, 377, L5

Bahcall, J. N., \& Spitzer, L. 1969, ApJ, 156, L63

Bechtold, J. 1987, in High Redshift and Primeval Galaxies, ed. J. Bergeron,

D. Kunth, R. Rocca-Volmerange, \& J. T. T. Van (Paris: Editions Frontières), 397

Bergeron, J., \& Ikeuchi, S. 1990, A\&A, 235, 8

Bergeron, J., \& Stasinska, G. 1986, A\&A, 169, 1

Black, J. H. 1981, MNRAS, 197, 533

Bond, J. R. 1990, in The Cosmic Background: 25 Years After, ed. R. Mandolesi \& N. Vittorio (Dordrecht: Kluwer), in press

Fall, S. M., Pei, Y. C., \& McMahon, R. G. 1989, ApJ, 341, L5

Fukugita, M., Futamase, T., \& Kasai, M. 1990a, MNRAS, 246, 25P

Fukugita, M., \& Hogan, C. 1990, Nature, 347, 120

Fukugita, M., Takahara, F., Yamashita, K., \& Yoshii, Y. 1990b, ApJ, 361, L1

Fukugita, M., \& Turner, E. L. 1991, MNRAS, 253, 17P

Gott, J. R., Park, M.-G., \& Lee, H. M. 1989, ApJ, 338, 1

Ikeuchi, S., Murakami, I., \& Rees, M. J. 1988, MNRAS, 236, 21 P

Ikeuchi, S., \& Ostriker, J. P. 1986, ApJ, 301, 522

Ikeuchi, S., \& Turner, E. L. 1991, ApJ, 381, L1

Jacoby, G. H., Ciardullo, R., \& Ford, H. 1990, ApJ, 356, 332

Kofman, L. A., \& Starobinsky, A. A. 1985, Soviet Astron., 11, 271

Kolb, E., \& Turner, M. S. 1989, The Early Universe (New York: AddisonWesley)
Lanzetta, K. M., et al. 1991, ApJS, 77, 1

Lu, L., Wolfe, A. M., \& Turnshek, D. A. 1991, ApJ, 367, 19

Mather, J. C., et al. 1990, ApJ, 354, L37

Morris, S. L., Weymann, R. J., Savage, B. D., \& Gilliland, B. I. 1991, ApJ, L21

Ostriker, J. P. \& Ikeuchi, S. 1983, ApJ, 263, L63

Rees, M. J. 1988, in QSO Absorption Lines: Probing the Universe, ed. J. L. Blades, D. A. Turnshek, \& C. A. Norman (Cambridge: Cambridge Univ. Press), 107

Sandage, A., \& Cacciari, C. 1990, ApJ, 350, 645

Sargent, W. L. W., Steidel, C. C., \& Boksenberg, A. 1988a, ApJS, 68, 539 1988b, ApJ, 334, 22
1989, ApJS, 69, 703

Steidel, C. C. 1990, ApJS, 72, 1

Steidel, C. C., \& Sargent, W. L. W. 1987, ApJ, 318, L11

Sugiyama, N., Gouda, N., \& Sasaki, M. 1990, ApJS, 74, 665

Tonry, J. L. 1991, ApJ., 373, L1

Trimble, V. 1987, ARA\&A, 25, 425

Turner, E. L. 1990, ApJ, 365, L43

Turner, M. S. 1991, preprint

Tytler, D. 1987, ApJ, 321, 69 